\title{
Design Study and Test Arrangement of HTS Transmission Line Power Cable for Fast Cycling Accelerator Magnets
}

\author{
Henryk Piekarz, Jamie Blowers, Steven Hays, Yuenian Huang and Vladimir Shiltsev
}

\begin{abstract}
Designs of the HTS transmission line power cable and the matching magnetic core for a fast-cycling accelerator dipole magnet are presented. The hysteretic and eddy currents induced power losses of the proposed HTS cable operating under various sweeping magnetic fields are projected and compared to those of the LTS cable in similar applications. The engineering design of the HTS power cable for the fast cycling dipole magnet is presented, and the test arrangement of a short-sample cable operating under the sweeping magnetic field is described.
\end{abstract}

Index Terms-HTS cable, magnetic core, AC power losses, synchrotrons.

\section{Motivation}

$\mathrm{T}$ HE fast-cycling synchrotrons are essential components of the accelerator systems future high energy particle physics programs [1,2,3]. Until now these type of synchrotrons utilized magnets powered with normal conducting cables. The large size of these conductor cables, necessary to carry the prescribed transport current, requires in turn large size of magnetic cores. As the current density in the superconductor is typically (100-150) times higher than that of the normal one the size of the superconducting power cable, and therefore the magnetic core, can be strongly minimized. In the fast-cycling operations the cryogenic power usage in superconducting magnets, however, is expected to be very high requiring large cryogenic plants with incurred long-term high operational costs offsetting the power system gains. The efforts [4] in designing fast-cycling accelerator magnets powered with the LTS (Low Temperature Superconductor, e.g. NbTi) cables did not succeed in bringing the cryogenic power usage to the level low enough that their application in a large-scale synchrotron would be cost effective. It has been suggested [5] that cables based on the HTS (High Temperature Superconductor) conductors, such as e.g. YBCO 344S-2G [6] which strands are wide but thin tapes consisting of only a single superconducting filament, may allow minimization of cryogenic power losses. The tape form of the YBCO strands allows for the parallel orientation of the filament's wide surface toward the magnetic field crossing the conductor and therefore exposing the narrow edge (d) of the strand to the strong parallel field $\left(B_{y}\right)$ while its

Manuscript received 16 October 2009. This work was supported by Fermi Research Alliance, LLC under DOE Contract DE-AC02-07CH11359.

H. Piekarz, J. Blowers, S. Hays, Y. Huang and V. Shiltsev are with Fermi National Accelerator Laboratory, Batavia, Il 60510, USA (phone: 630-8402105; fax: 630-840-8036; e-mail: hpiekarz@fnal.gov ). wide width (w) faces only the weak perpendicular field $\left(\mathrm{B}_{\mathrm{x}}\right)$. This arrangement will reduce the conductor power losses by the product of $\left(<\left|B_{x}\right|>/ B_{y}\right) \times(w / d)$. In practice, however, it is difficult to arrange a strict parallel orientation of the strands toward the $\mathrm{B}_{\mathrm{y}}$ field, so the losses will be always higher than in the ideal case. Nevertheless we expect them to be significantly lower than for the LTS type conductors in similar applications.

\section{HTS CABLE AND MAGNETIC CORE DESIGNS}

A much simplified view of the $344 \mathrm{~S}-2 \mathrm{G}$ strand is shown in figure 1 together with the directions of the transport current and the sweeping external magnetic field. There are three main strand components: (1) superconductor filament (YBCO, $2 \mu \mathrm{m}$ ), (2) magnetic substrate (Ni5\%W, $80 \mu \mathrm{m}$ ) and (3) strand holding lamination $(\mathrm{Cu}, 2 \times 50 \mu \mathrm{m})$. The transport current, $\mathrm{I}_{\mathrm{t}}$, which flows along the strand length generates self-field, $\mathrm{B}_{\text {self }}$. The $\mathrm{x}$-component of the external $\mathrm{B}$-field, $\mathrm{B}_{\mathrm{x}}$, generates screen currents, $\mathrm{J}_{\mathrm{s}}$, which can alter the field pattern [7] within the filament space affecting in this way the strand mean critical current. If the strands are not electrically isolated then this effect gives rise to the transport current coupling between the neighboring strands inducing in this way power loss along the

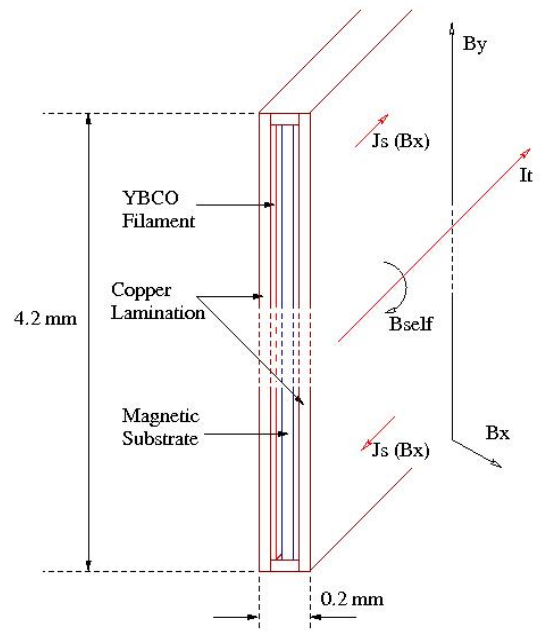

Fig. 1. The main components of the $344 \mathrm{~S}-2 \mathrm{G}$ strand with indications of the transport current, the self-field, the external sweeping magnetic field (By, Bx), and the Bx induced screen currents, Js.

entire cable length. If the strands are isolated then the screen currents induced power loss is passed on to the cable ends outside the magnet gap and typically into the conductor splice areas. In addition to above a possible variation of the transport 
current in the individual strands may also lead to the self-field induced coupling which in turn causes the power loss as well.

A conceptual arrangement of the 344S-2G strands to form a power cable is shown in figure 2 . The strands are electrically isolated from each other with staggered Kapton rings. These

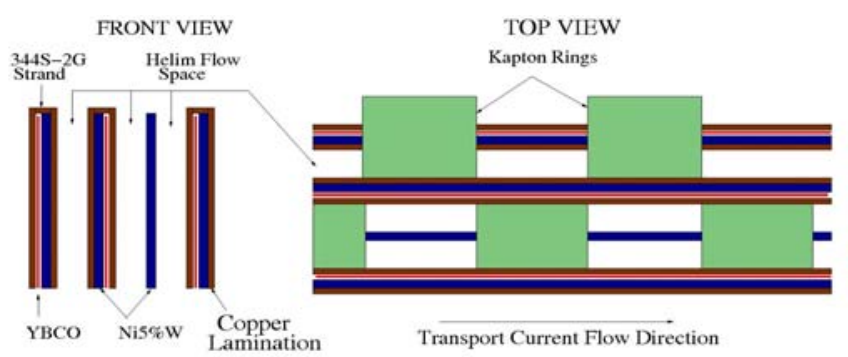

Fig. 2. Conceptual arrangement of 344S-2G strands to construct a cable

rings also reserve helium flow space between the strands allowing for $50 \%$ of the strand surface to be in a direct contact with helium coolant. The strands are arranged in pairs in a way that their magnetic substrates face each other. As the transport current direction is the same in all strands the self fields circulate in the opposite directions within the each other substrate space. This helps to minimize the self-field coupling [8] up to the Ni5\%W saturation field of $\sim 200 \mathrm{G}$. With an additional substrate tape placed between the strand's pairs the self-field coupling in the entire cable is expected to be much reduced.

We will use the DSFMR [1] and the RCS [2] accelerator proposals to present examples of the HTS power cable design for the fast cycling magnets. We assume the magnet cable to operate at $5 \mathrm{~K}$ with a transport current, $\mathrm{I}_{\mathrm{t}}$, at $\sim 75 \%$ of the cable critical current, $I_{c}$ at $15 \mathrm{~K}$. This means that if the number of strands is based on $I_{c}$ at $15 \mathrm{~K}$ then the allowable operational temperature margin is $10 \mathrm{~K}$. In the $(0-2) \mathrm{T}$ range with the parallel field orientation to the wide surface of the 344S-2G strands the critical current density is $\sim 1050 \mathrm{~A} / \mathrm{mm}^{2}$ at $5 \mathrm{~K}$, and $\sim 910 \mathrm{~A} / \mathrm{mm}^{2}$ at $15 \mathrm{~K}$ [9]. The number of the required strands for the DSFMR and RCS magnet cables (two per magnet gap) is then 192 and 48, respectively. The main parameters of the DSFMR and the RCS magnets are listed in Table 1.

TABLE I DSFMR AND RCS MAGNET PARAMETERS

\begin{tabular}{cccccc}
\hline \hline Accelerator & $\begin{array}{c}\text { Magnet gap } \\
\text { H[mm] x V[mm] }\end{array}$ & $\begin{array}{c}\mathbf{B} \\
{[\mathbf{T}]}\end{array}$ & $\begin{array}{c}\mathbf{d B} / \mathbf{d t} \\
{[\mathbf{T} / \mathbf{s}]}\end{array}$ & $\begin{array}{c}\mathbf{I} \\
{[\mathbf{k A}]}\end{array}$ & $\begin{array}{c}\mathbf{N} \\
\text { strands }\end{array}$ \\
\hline DSFMR & $80 \times 40$ & 2 & 2 & 70 & 192 \\
RCS & $100 \times 44$ & 0.5 & 8 & 18 & 48 \\
\hline \hline
\end{tabular}

The designs of both the HTS cable and the magnetic core are aimed at minimization of the $\mathrm{B}_{\mathrm{x}}$ field component crossing the conductor stack. In the central (vertical and horizontal) point of the cable the $B_{x}$ is zero but it rises for the higher (lower) vertical stack positions in the cable. Our studies found that the closer the top/bottom of the conductor stack extends to the wall of cable cavity in the core the more parallel are the Bfield lines within the conductor space, and so the lower is the mean $</ \mathrm{B}_{\mathrm{x}} />$ value. The optimized design of the DSFMR core with the power cable is shown in figure 3 . The magnetic core is constructed using $100 \mu \mathrm{m}$ thick Fe3\%Si steel laminations to strongly reduce core eddy losses and improve B-field quality.

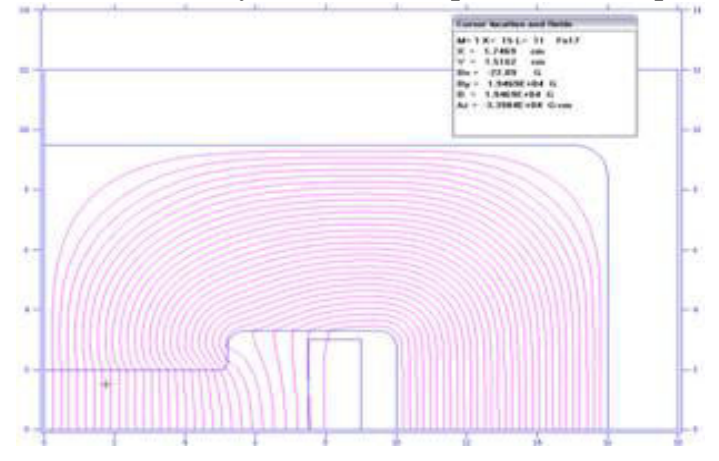

Fig. 3. Core design and conductor placement for DSFMR magnet

In order to spread the HTS strands as much as possible evenly within the cable space we arranged them into six subcables, each with 16 strands. A conceptual view of a sub-cable is shown in figure 4 , and the arrangement of the magnet cable is shown in figure 5 . The conductor stack is placed inside the

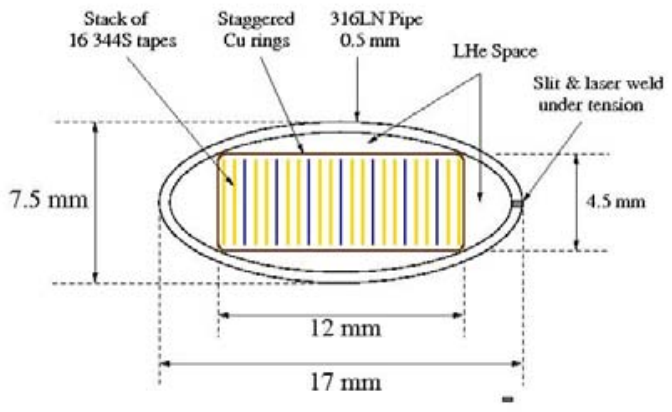

Fig. 4. Conceptual view of conductor stack with cryogenic pipe

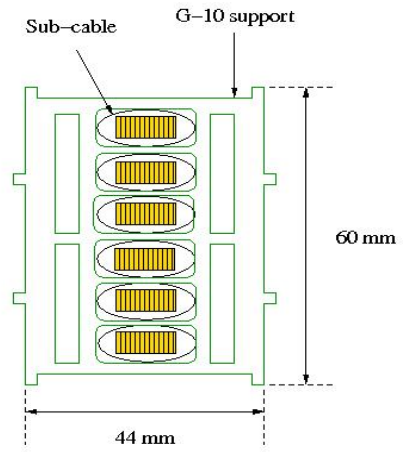

Fig. 5. Arrangement of DSFMR six sub-cables in a G10 ring holder.

elliptical cold pipe which is slit on one side along its entire length. This shape of the pipe allows placing the sub-cable close to the top (bottom) wall of the core. The elliptical shape also prevents stack from twisting induced by the magnetic forces, and it helps to secure its orientation with respect to the magnetic field. The conductor stack is tightly wrapped with staggered copper rings which are soldered together using a low-temperature tin to avoid damage of the strands. The pipe with the inserted conductor stack is laser welded under 0.5 
Ton/m tension to immobilize the stack. The sub-cables are held together using staggered (3/meter) G-10 rings (figure 5). As only the pegs of these rings are in contact with the magnet core the cable is well insulated but the cryostat pipe must encompass the entire magnet assembly. The arrangement of the DSFMR magnet pair in the accelerator ring is shown in figure 6 . The cores are cooled to $77 \mathrm{~K}$ using liquid nitrogen to

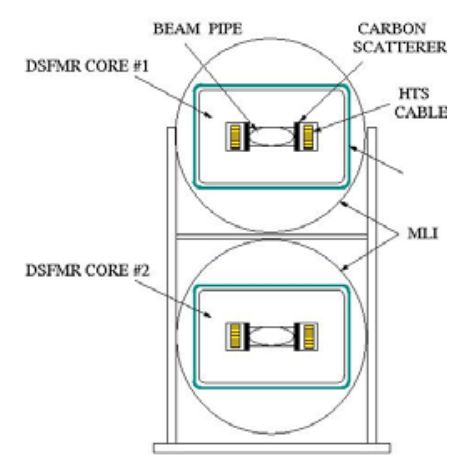

Fig. 6. Arrangement of DSFMR magnets and power cable cryostats

minimize environmental radiation heat load to the cable, and thus strongly minimizing in this way the required cable helium cooling power. This is in contrast to the LTS powered fastcycling magnets where the core is typically cooled down to 4 $\mathrm{K}$ [4] to facilitate operations of the cable with very narrow allowable temperature margin, typically $<2 \mathrm{~K}$.

\section{ESTIMATED POWER LOSSES FOR DSFMR AND RCS ACCELERATOR MAGNETS}

In order to estimate the power losses with the HTS cable we first determine the $d</ B_{x} />/ d t$ rate range of interest. With the parallel orientation of the HTS tapes toward $\mathrm{B}_{\mathrm{y}}$ field the average $</ B_{x} />$ field crossing the cable as shown in figure 5 is $\sim 300 \mathrm{G}$ (for comparison in the magnet beam gap it is $\sim 25 \mathrm{G}$ ). In the two most top/bottom sub-cables the $\left\langle\mathrm{B}_{\mathrm{y}}\right\rangle$ field lines are inclined, however, by $\sim 2.5^{\circ}$ with respect to the HTS tapes. As a result the effective $\left\langle/ \mathrm{B}_{\mathrm{y}} />\right.$ field component in these stacks is about a factor 2 stronger than the $\left\langle/ \mathrm{B}_{\mathrm{x}} />\right.$ one. Using the $</ \mathrm{B}_{\mathrm{y}} />$ instead of the $\left\langle/ B_{\mathrm{X}} />\right.$ for these sub-cables leads to the effective $</ \mathrm{B}_{\mathrm{x}} />$ of $\sim 475 \mathrm{G}$. On the other hand if the HTS stacks in those two sub-cables were arranged to be parallel to their local $\mathrm{B}_{\mathrm{y}}$ field, the $\left\langle/ \mathrm{B}_{\mathrm{x}} />\right.$ value of the full cable would be reduced to $\sim 175 \mathrm{G}$. Based on the above we conclude that for the $0.5 \mathrm{~Hz}$ cycle of DSFMR magnet operation one may expect effective $\mathrm{d}</ \mathrm{B}_{\mathrm{x}} />/ \mathrm{dt}$ rate to be between $0.02 \mathrm{~T} / \mathrm{s}$ and $0.05 \mathrm{~T} / \mathrm{s}$.

In the fast-cycling operations the power losses come from 3 sources: (1) power cable, (2) magnet core and (3) beam tube. The power loss in the cable is by far the largest one and the most likely one to affect the magnet performance. The power losses in the cable involve both the hysteretic and the eddy currents ones while the core and the beam tube are due to eddy currents only. In the 344S-2G strand the power losses of the YBCO filament are hysteretic, in the magnetic substrate are both hysteretic and eddy currents, and the power loss in the $\mathrm{Cu}$ lamination is due to eddy currents. The hysteretic losses scale with $d B / d t$ rate while the eddy ones scale with $(d B / d t)^{2}$. This means that with an increase of the magnetic field rate the power loss contribution from the components other than YBCO filament may become dominant. Power losses of the YBCO coated strands were thoroughly analyzed in [10] and very preliminary projections of these losses for the DSFMR type magnet cable were presented in [5]. In this paper we apply the analysis from [10] to the new HTS cable design, as described in Section II.

A summary of the projected hysteretic and eddy current losses for the 344S-2G strand components at $d</ \mathrm{B}_{\mathrm{x}} />/ \mathrm{dt}=0.03$ $\mathrm{T} / \mathrm{s}$ cycling rate is given in Table II, and projections of total hysteretic and eddy losses up to $\mathrm{d}</ \mathrm{B}_{\mathrm{x}} />/ \mathrm{dt}=0.1 \mathrm{~T} / \mathrm{s}$ range are shown in figure 7 . We observe that the YBCO hysteretic loss

TABLE II POWER LOSSES OF 344S-2G STRAND (0.03 T/s)

\begin{tabular}{lcc}
\hline \multicolumn{1}{c}{ Strand component } & P-hyst $[\mathbf{m W} / \mathbf{m}]$ & P-eddy $[\mathbf{m W} / \mathbf{m}]$ \\
\hline YBCO, $2 \mu \mathrm{m}$ & 6 & - \\
Ni5\%W, $120 \mu \mathrm{m}$ & $310^{-3}$ & 0.24 \\
Cu lamination $100 \mu \mathrm{m}$ & - & 0.40 \\
P-hyst total & 6 & - \\
P-eddy total & - & 0.64 \\
\hline \hline
\end{tabular}

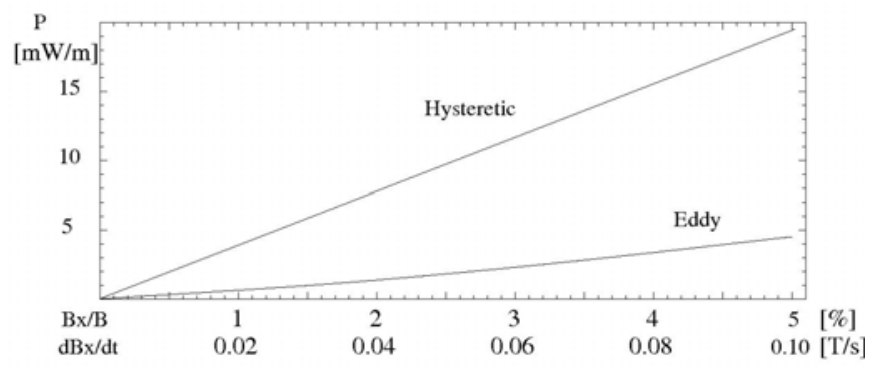

Fig. 7. Projected hysteretic and eddy power loss of the 344S-2G strand as a function of $d</ B_{x} />/ d t$ rate.

is strongly dominant and the eddy loss would match them only at $\mathrm{d}</ \mathrm{B}_{\mathrm{x}} />/ \mathrm{dt}$ rate of $\sim 0.3 \mathrm{~T} / \mathrm{s}$. At the expected $\mathrm{d}</ \mathrm{B}_{\mathrm{x}} />/ \mathrm{dt}$ rate of $0.03 \mathrm{~T} / \mathrm{s}$ the DSFMR magnet cable total power loss is $192 \mathrm{x}$ $6.64 \mathrm{~mW} / \mathrm{m}=\sim 1.3 \mathrm{~W} / \mathrm{m}$. For comparison, the cable power loss of the $2 \mathrm{~T} / \mathrm{s}$ magnet based on the LTS conductor is $\sim 12$ $\mathrm{W} / \mathrm{m}$ [4], thus about an order of magnitude higher. For the RCS magnet with 4 times lower B-field but 16 times higher cycling rate we tentatively project total strand power loss of $28 \mathrm{~mW} / \mathrm{m}$, leading to $48 \times 28 \mathrm{~mW} / \mathrm{m}=\sim 1.3 \mathrm{~W} / \mathrm{m}$ for the magnet cable, same as for the DSFMR magnet design. As shown in [4] the eddy and the hysteretic losses in the magnet yoke (1mm laminations @ $4.2 \mathrm{~K}$ ) increase the total magnet power loss by $60 \%$. The $4.2 \mathrm{~K}$ temperature of the core allows minimization of both coil size and the core and also helping to operate the cable with very narrow allowable temperature margin. As our cable design allows for $10 \mathrm{~K}$ margin we expect cooling of the core to $77 \mathrm{~K}$ to be sufficient, and we also use $0.1 \mathrm{~mm}$ laminations. This arrangement makes magnetic core power losses negligible (relative to cable). Consequently, for the DSFMR accelerator (dual ring of $6280 \mathrm{~m}$ circumference) 
the total projected power loss for $2 \mathrm{~T} / \mathrm{s}$ cycling rate is $\sim 16 \mathrm{~kW}$, and for the RCS machine (560 m circumference) the total projected power loss at $8 \mathrm{~T} / \mathrm{s}$ cycling rate is $\sim 0.8 \mathrm{~kW}$.

\section{TEST HTS CABLE ENGINEERING DESIGN}

The test HTS cable consists of $12140 \mathrm{~cm}$ long 344S-2G strands and $5124 \mathrm{~cm}$ long and $80 \mu \mathrm{m}$ thick Ni5\%W tapes between the HTS pairs. The substrate tapes are slightly shorter to be isolated from the strands in the splice areas. The strands are arranged as described in Section II, and then placed inside the $1 / 2$ " 316LN slit elliptical pipe. After securing proper orientation of the stack the slit pipe is laser welded under tension. The connection of the stack with the power lead is shown in figure 8 . The splice copper block is tightly embedded in the Teflon ring which separates helium flow in the conductor section from the flow in the lead. The lead is made of $7160 \mathrm{~cm}$ long, $1 / 4$ " in diameter copper rods. Multiple

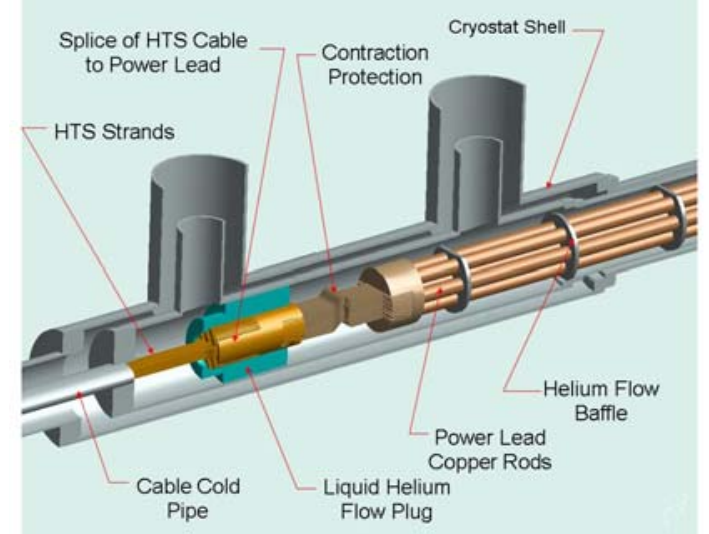

Fig. 8. Connection of the HTS cable with conventional power lead

baffles on the lead rods assembly force the liquid helium to flow interchangeably through top and bottom space of the lead along its entire length. The splice block and the lead end are connected using a stack of the oversized and flexible copper tapes to counter the contraction and the expansion of the leads and the HTS cable during cool-downs and warm-ups.

\section{HTS CABLE TEST ARRANGEMENT}

The HTS cable test arrangement is shown in figure 9. The total conductor loop is $9 \mathrm{~m}$ long and it consists of: (1) $1.4 \mathrm{~m}$ test section, (2) two $1.6 \mathrm{~m}$ leads, and (3) $5 \mathrm{~m}$ long return (copper) conductor. The test cable section passes through the center of a $1.2 \mathrm{~m}$ long dipole magnet (CDA) of $0.5 \mathrm{~T}$ maximum B-field. The magnet is set to operate up to $6 \mathrm{~Hz}$ generating a sweeping $\mathrm{dB} / \mathrm{dt}$ field of $6 \mathrm{~T} / \mathrm{s}$. The clamps at the power leads warm ends allow rotation of the test cable (with leads) to expose the wide side of the HTS stack to a varying strength of $B_{y}$ magnetic field. The conductor loop is closed with the $10 \mathrm{kA}$ current source which is used only to measure the di/dt transport current power losses. For these measurements the CDA dipole will be removed. The HTS cable section is cooled with supercritical helium supplied from the E4R plant while the leads are cooled with liquid helium supplied from $500 \mathrm{l}$ dewars. The test cable section is instrumented with multiple high precision temperature

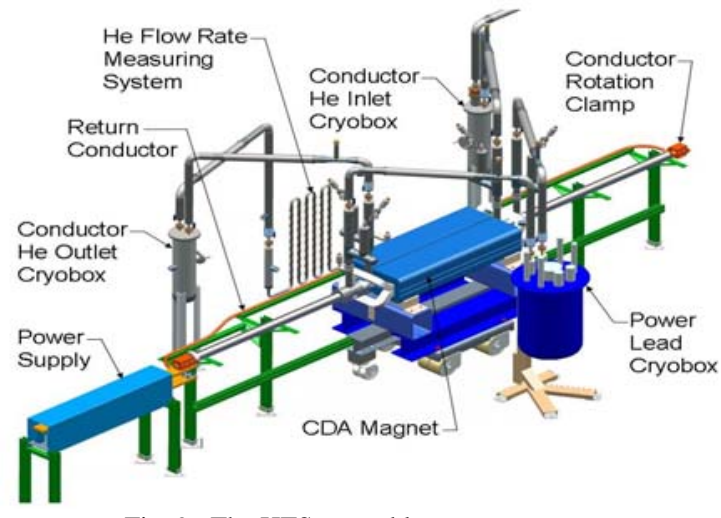

Fig. 9. The HTS test cable arrangement

sensors as well as the liquid helium flow rate and pressure drop measuring systems.

\section{SUMMARY AND CONCLUSIONS}

We believe there is a reasonable prospect for using the HTS tape strands to construct the power cables for fast-cycling accelerator magnets. The arrangement of the HTS strand stack into a cable requires high precision mechanical work to secure the stack in proper orientation with respect to the B-field, and also to ensure mechanical sustainability in long term operations. The forthcoming test is set to examine both the power losses as a function of the $\mathrm{dB} / \mathrm{dt}$ rate, as well as the ability of precision alignment of the HTS tapes with respect to the B-field in the cable space.

\section{REFERENCES}

[1] H. Piekarz, S. Hays, Y. Huang and V. Shiltsev, "Design Considerations of Fast-Cycling Synchrotrons Using Superconducting Transmission Line Magnets”, European Particle Accelerator Conference EPAC-08 Genoa, 23-27 June 2008

[2] V. Lebedev, "RCS for Project $X$ " (unpublished) Project X Collaboration Meeting, September, 2009, Fermilab

[3] L.Bottura, R. Maccaferri, C. Maglioni, V. Parma, L. Rossi, G. de Rijk, W. Scandale, L. Serio, D. Tommasini, "A Conceptial design study for a super-ferric PS2” (unpublished), GLM, CERN, October 8, 2007

[4] A. Stafiniak, C. Schroeder, E. Floch, P. Schnizer, H. Mueller, E. Fisher, G. Moritz, F. Marzouki, F. Walter, M. Kauschke, J. Kaugarts, "Cold Testing of Rapidly-Cycling Model Magnets for SIS 100 and SIS $300-$ Methods and Results", WAMSDO Workshop, CERN-2009-001, 19-23 May, 2008

[5] H. Piekarz, S. Hays, Y. Huang, G. de Rijk, L. Rossi, "Design Considerations for Fast-Cycling Superconducting Accelerator Magnet of 2 Tesla B-field Generatedby Transmission Line Conductor of up to 100 kA Current", Int. Conf. on Magnet Technology MT-20, Philadelphia, August 27-31, 2007

[6] X. Li, et al."Development of Second Generation HTS Wire at American Superconductor", IEEE, 19, 3231-3235 (2009)

[7] M. N. Wilson, "Superconducting Magnets” ISBN 019 854810 9, 1997

[8] O. Tsukamoto et al., "AC Loss Characteristics of Stacked Conductors Composed of HTS Coated Conductors with Magnetic Substrates", IEEE 17, 3195-3197, 2007

[9] D. Turioni, E. Barzi, M. Lamm, V. Lombardo, C. Thieme, A. V. Zlobin, "Angular Measurements of HTS Critical Current for High Field Solenoids”, AIP Conf. Proc. 986, 45 (2008)

[10] M. D. Sumption, E. W. Collings, P. N. Barnes, “AC loss in stripped (filamentary) YBCO coated conductors leading to designs for high frequencies and field-sweep amplitudes", Supercondctor.Science Technology, 18, 122-134 (2005) 